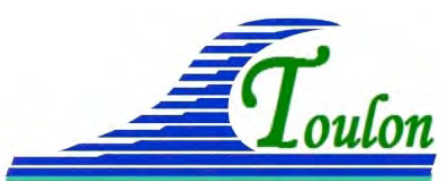

XIV èmes Journées Nationales Génie Côtier - Génie Civil Toulon, 29 juin au $1^{\text {er }}$ juillet 2016

DOI:10.5150/jngcgc.2016.049 (C) Editions Paralia CFL

disponible en ligne - http://www.paralia.fr - available online

\title{
Conception d'une couche filtre granulaire dynamiquement fermée au pied des digues de la Nouvelle Route du Littoral à la Réunion
}

\author{
David LAJOIE ${ }^{1}$ \\ 1. Ingénieur Conseil, SAS HydroGC, 06323656 92, 06530 Le Tignet, France. \\ david.lajoie@hydrogc.fr
}

\section{Résumé :}

La Nouvelle Route du Littoral à la Réunion est en partie protégée par des digues à talus classiques, dont la profondeur du pied varie de $5 \mathrm{~m}$ à $10 \mathrm{~m}$ environ. Le pied de l'ouvrage est ensouillé dans le fond sableux et prolongé par un tapis de blocs, le tout offrant une stabilité à l'ensemble du talus en cas d'affouillement significatif. Le pied de l'ouvrage a été conçu en intégrant une membrane géotextile, empêchant les infiltrations de sable qui provoqueraient des tassements locaux susceptibles de se répercuter sur l'infrastructure routière. La pose de géotextile est difficile en milieu fréquemment agité par les houles d'alizés, si bien qu'une adaptation de type filtre granulaire a été étudiée dans le cadre des études d'exécution d'Entreprises. La conception classique, basée sur l'application des règles usuelles de fermeture géométrique des filtres, conduisait à superposer plusieurs couches de granulométries différentes, peu compatibles avec les contraintes réelles du chantier maritime. Une alternative de type monocouche granulaire de grande épaisseur a pu être conçue, en se référant aux avancées récentes sur les notions de fermeture dynamique des filtres. La conception s'appuie également sur le principe d'un tri naturel des grains à l'intérieur de la couche géométriquement ouverte, aboutissant à terme à une configuration stable après tassement très modéré.

Mots-clés : Digue à talus, Filtre granulaire, Fermeture dynamique.

\begin{abstract}
:
Geotextile filters at the toe of the breakwater of the New Coastal Road are rather difficult to lay down because of depth and current wave climatology. Classical alternatives based on granular filter involve several layers of coarse sand and gravels above the natural fine sandy bottom, which are not suitable for the maritime construction site constraints. Another alternative is designed, based on a single and thick layer of mixed sand and gravels. Recent researches about dynamically closed filters are used. Natural winnoning of the smallest sand grains from the geometrically open filter is also used to justify the long term stability of the granular filter.
\end{abstract}

Keywords: Breakwaters, Granular filters, Dynamic closure. 


\section{Introduction}

La nouvelle route du littoral doit relier Saint-Denis de la Réunion à la Possession, offrant un linéaire élargi et sécurisé au regard des risques de chutes de pierres venant de falaises adjacentes. L'infrastructure routière est composée de terre-pleins gagnés sur la mer ou de tronçons de type viaduc. Les parties remblayées sont protégées par des digues à talus classiques, dont la profondeur du pied varie de $5 \mathrm{~m}$ à $10 \mathrm{~m}$. La carapace, soumise potentiellement à des houles cycloniques, est constituée de blocs artificiels de type Accropodes II ou X-blocs, dont le pied est buté par des enrochements. Le pied de l'ouvrage est ensouillé dans le fond sableux et prolongé par un tapis de blocs, le tout offrant une stabilité à l'ensemble du talus en cas d'affouillement significatif.

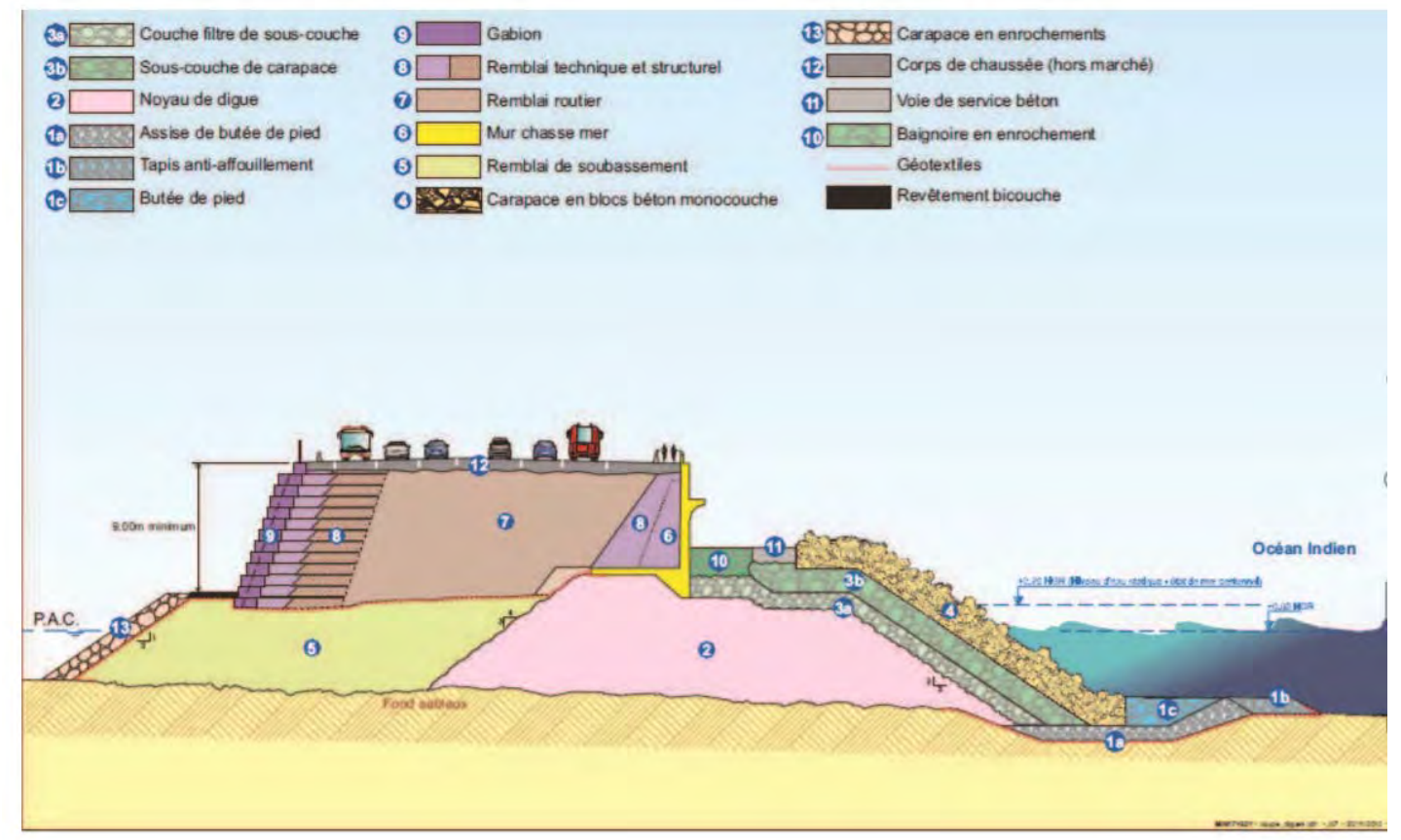

Figure 1. Une des coupes types des digues de protection de la NRL (source CCTP, EGIS).

Le pied de l'ouvrage a par ailleurs été conçu par le bureau d'étude EGIS, Maître d'œuvre, pour éviter des poinçonnements potentiels de blocs de sous-couche ou des infiltrations de sable qui provoqueraient des tassements locaux susceptibles de se répercuter sur l'infrastructure routière. La solution de base prévoyait la pose d'une membrane géotextile sous la butée de pied pour empêcher la remontée des sables fins. Cette disposition, certes classique et justifiée par l'état de l'art, a été jugée difficile à mettre en œuvre par le groupement d'entreprises GTOI- SBTPC-VINCI, chargé de la construction de l'ouvrage. Il semblait délicat de respecter une certaine qualité de pose de géotextile, par $10 \mathrm{~m}$ de fond, dans une zone régulièrement soumise aux houles d'alizés. 


\section{XIV $V^{\text {èmes }}$ Journées Nationales Génie Côtier - Génie Civil Toulon, 29 juin au $1^{\text {er }}$ juillet 2016}

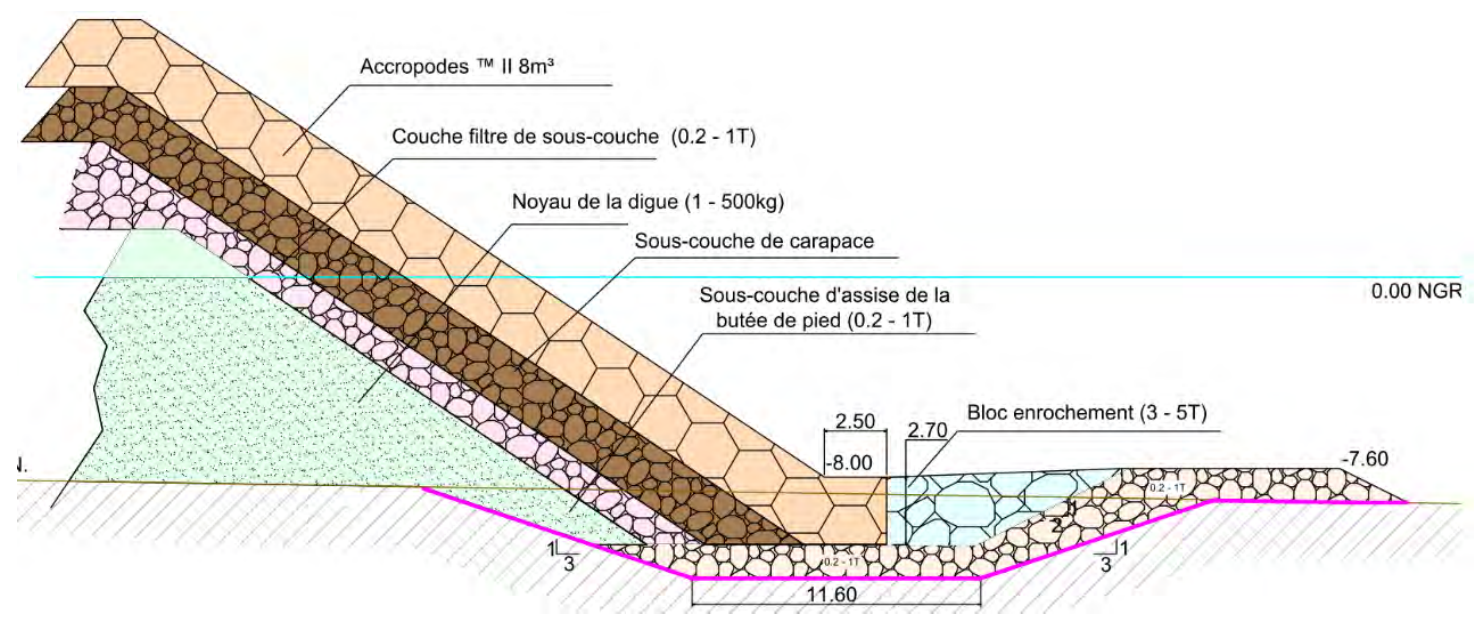

Figure 2. Coupe type du pied de digue en solution de base avec géotextile.

Une alternative de type couche filtre granulaire de conception originale a été recherchée pour respecter les propriétés filtrantes des géotextiles, tout en s’adaptant aux méthodes de construction, pour lesquelles il est préférable d'éviter la pose de plusieurs couches successives de granulométrie variable.

\section{Hypothèses de conception et de construction}

Le pied de digue repose sur un sol sableux à graveleux. Certaines zones contiennent du sable fin, dont les diamètres passant $\mathrm{D}_{15}-\mathrm{D}_{50}-\mathrm{D}_{85}$ ont pour valeurs $(80-200-430 \mu \mathrm{m})$. La butée de pied des blocs artificiels est composée d'enrochements 3-5t, reposant sur une sous-couche de blocs 0.2-1 t. Ces derniers sont utilisés en première sous-couche du noyau de tout-venant, mais aussi en tant que tapis anti-affouillement.

Les méthodes de construction impliquent du matériel spécifique et lourd pour draguer toutes les souilles en pied en quelques semaines. Les souilles draguées sont temporairement protégées par les enrochements $0.2-1 \mathrm{t}$, pendant toute la phase travaux. Les souilles sont recouvertes progressivement par la butée de pied, au gré de l'avancement de la construction de la digue. Les blocs 0.2-1 t ont été conçus pour offrir suffisamment de stabilité face aux conditions cycloniques, avec en appui les essais en canal menés par le Maître d'œuvre. La taille des blocs 0.2-1 t ne saurait toutefois être réduite, ou étendue vers des granulométries plus petites.

\section{Etat de l'art sur les couches filtres granulaires}

L'application de filtres granulaires est l'alternative classique pour respecter la fonction de rétention des sables fins au niveau du pied de talus, sous les blocs 0.2-1 t. Les règles de l'art en matière de conception des couches filtres sont fondées sur les trois critères suivants : 
i. Critère de rétention : les matériaux fins de la base ne doivent pas pénétrer la couche filtre. Le rapport entre le diamètre $D_{15}(f)$ des petits grains du filtre et le diamètre $\mathrm{D}_{85}$ (b) des gros grains de la base doit être tel que (critère de Terzaghi) :

$$
\frac{D_{15}(f)}{D_{85}(b)}<5
$$

ii. Critère de stabilité interne : les petits grains d'une couche de matériaux ne doivent pas migrer à travers les vides de cette même couche. Cela se traduit par un rapport limité entre diamètres passants, dont une expression simple et généralisée a été donnée par Pylarczyk :

$$
\frac{D_{60}}{D_{10}}<10
$$

iii. Critère de perméabilité : le filtre doit avoir une perméabilité suffisante par rapport à la base pour ne pas résister aux écoulements hydrauliques et risquer de créer des pressions interstitielles :

$$
\frac{D_{15}(f)}{D_{15}(b)}>4
$$

En dessous de la couche filtre, les plus gros grains du sable fin ayant un diamètre de l'ordre de $D_{85} \approx 0.4 \mathrm{~mm}$, la taille des plus petits grains du filtre granulaire doit être inférieure à $D_{15} \approx 0.4 \mathrm{~mm} \times 5=2 \mathrm{~mm}$. Au-dessus de la couche filtre, les plus petits blocs 0.2 - $1 \mathrm{t}$ ont un diamètre $D_{15} \approx 550 \mathrm{~mm}$. La taille des plus gros grains du filtre granulaire doit être supérieure à $D_{85}=550 \mathrm{~mm} / 5=110 \mathrm{~mm}$.

L'étendue des valeurs $\mathrm{D}_{15}<2 \mathrm{~mm}$ et $\mathrm{D}_{85}>110 \mathrm{~mm}$ permet certes de respecter les critères de rétention entre les couches, mais pas le critère de stabilité interne du filtre granulaire, le rapport $D_{85} / D_{15} \approx 55$ étant incompatible avec le respect du critère $D_{60} / D_{10}<10$.

Si le critère de stabilité interne était respecté, le filtre serait dit géométriquement fermé. Pour obtenir un filtre géométriquement fermé entre les blocs $0.2-1 \mathrm{t}$ et le sable fin, il faudrait appliquer deux couches distinctes, par exemple 2-20 mm, puis 60-150 mm.

L'application de deux couches distinctes, de l'ordre de $2 \times 30 \mathrm{~cm}$ d'épaisseur, si elle est théoriquement largement acceptable, ne cadre pas avec les exigences réelles d'un chantier maritime, à la fois au regard de la facilité de tri en carrière, puis au regard de la précision de la pose des couches successives. Les différentes méthodes de travaux maritimes en milieu agité tendent à privilégier les solutions impliquant la pose d'une seule couche, avec une certaine épaisseur. Une alternative à la solution bicouche granulaire a été recherchée dans ce sens, s'appuyant sur la notion de filtre géométriquement ouvert, mais dynamiquement fermé. 


\section{XIV èmes Journées Nationales Génie Côtier - Génie Civil \\ Toulon, 29 juin au $1^{\text {er }}$ juillet 2016}

\section{Notion de filtre fermé dynamiquement}

Une couche de matériau meuble peut être géométriquement ouverte (rétention ou érosion interne non respectée), mais dynamiquement stable, c'est-à-dire que l'épaisseur de matériau la recouvrant est suffisamment épaisse pour dissiper l'écoulement turbulent et réduire les contraintes à même de la déstabiliser.

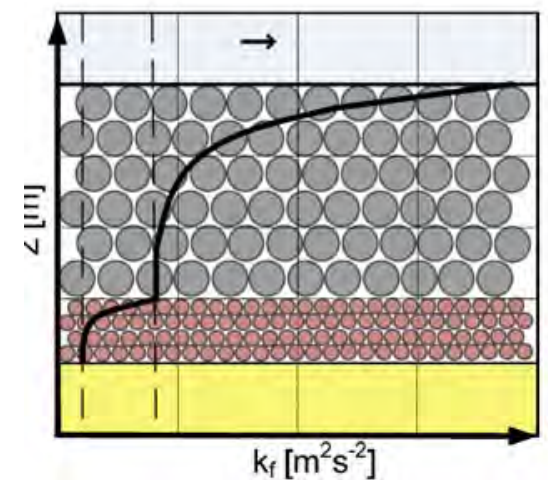

Figure 3. Principe de la dissipation d'énergie cinétique turbulente à travers les couches successive (VAN DE SANDE, 2013).

Des formulations spécifiques inhérentes aux filtres ouverts sous l'action de courants horizontaux de fond ont été conduites, impliquant l'épaisseur de couche filtre supérieure nécessaire pour fermer dynamiquement une couche base inférieure. Il importe que l'épaisseur de couche filtre protectrice soit suffisante pour que les matériaux de la couche base soient aussi stables que ceux de la couche filtre. HOFFMANS (2012), puis VAN DE SANDE (2013), ont établi une formulation permettant d'estimer cette épaisseur.

$\frac{d}{D_{50 f}}=\alpha_{d} \ln \left(\frac{\Delta_{f}}{\Delta_{b}} \frac{D_{50 f}}{D_{50 b}} \frac{\Psi_{f}}{\Psi_{b}} \frac{\left(1-\gamma \mathrm{V}_{G f}\right)}{\left(1-\gamma \mathrm{V}_{G b}\right)}\right)$

avec :

d : épaisseur nécessaire de couche filtre (f) pour fermer dynamiquement la base (b)

$\Delta$ : densité relative du matériau

$\Psi$ : paramètre critique de Shields $\approx 0.055$ pour des diamètres $>25 \mathrm{~mm}$

$\mathrm{V}_{G}=1-\frac{D_{15}}{D_{50}}=$ coefficient de variation de la distribution de la taille de grains

$\gamma=0.625$

$D_{15 f}$ : diamètre passant à $15 \%$ du volume de la couche filtre $f$

$\alpha_{d}=1.2$

La formulation ne fait pas intervenir directement la contrainte de sollicitation sur la couche filtre supérieure. Elle est fondée sur le rapport relatif $\eta$ des contraintes de cisaillement $\tau_{f}$ et $\tau_{b}$ sollicitant simultanément le filtre et la base, tel que : 


$$
\eta=\frac{\tau_{b}}{\tau_{f}}=\frac{\Delta_{b}}{\Delta_{f}} \frac{D_{50 b}}{D_{50 f}} \frac{\Psi_{b}}{\Psi_{f}} \frac{\left(1-\gamma \mathrm{V}_{G b}\right)}{\left(1-\gamma \mathrm{V}_{G f}\right)}=e^{\frac{-d}{\alpha_{d} D_{50 f}}}
$$

L'équation (4), avec un coefficient d'atténuation théorique $\alpha_{\mathrm{d}}=1.2$, majore les résultats d'essais en laboratoire menés par Van De Sande, dont les conclusions sont les suivantes :

- la forme logarithmique de l'évolution de l'épaisseur d est confirmée ;

- la valeur du facteur $\alpha_{\mathrm{d}}=1.2$ est très conservative, l'analyse des essais aboutissant à $\alpha_{d}=0.46$ : en moyenne, $\alpha_{d}=0.69$, comme valeur majorante à $90 \%$ de confiance, $\alpha_{d}=0.82$ comme valeur majorante maximale.

L'approche théorique et expérimentale est fondée sur des sollicitations de courants turbulents, et pas de houles proprement dites, avec vitesses oscillantes. Les houles cycloniques entraînent toutefois des oscillations horizontales longues des particules fluides au fond, dont les effets d'inertie sont faibles devant les effets de traînée. L'approche, fondée sur l'écart relatif entre les contraintes de traînée entre couches et l'atténuation des courants par dissipation à travers les couches, n'est pas fondamentalement incompatible avec les sollicitations cycloniques occasionnant des trajectoires de courants longues devant la taille des blocs. Le raisonnement ci-dessus, fondé sur le rapport des contraintes tangentielles entre couches, resterait valable dans la mesure où la dissipation d'énergie à travers la couche filtre est la même pour un courant continu que pour un courant orbital de houle. Hoffmans valide l'application de sa formule à des courants orbitaux de houle, le rapport de laboratoire (VAN DE SANDE, 2013) étant toutefois plus circonspect, mettant en garde contre l'usage de cette formule pour des courants très turbulents. Les courants descendants de la carapace après déferlement sont également sources de forts gradients hydrauliques et donc de turbulence. Dans le cas des sollicitations de houle, l'emploi de la formule ci-dessus est donc encore à parfaire, l'usage du coefficient très majorant $\alpha_{\mathrm{d}}=1.2$ étant un gage de sécurité pour cerner les bons ordres de grandeur.

\section{Stabilité d'un filtre géométriquement ouvert}

La théorie ci-dessus est exploitée pour concevoir un filtre géométriquement ouvert dont la granulométrie théorique est telle que $\left(\mathrm{D}_{15}-\mathrm{D}_{50}-\mathrm{D}_{85}\right)=(2-25-150 \mathrm{~mm})$. Le filtre est placé sous :

- 2 couches d'enrochements 3-5 t, d'épaisseur $2.7 \mathrm{~m}$

- 2 couches d'enrochements $0.2-1 \mathrm{t}$, d'épaisseur $1.3 \mathrm{~m}$

Les diamètres passant de tamisage ont été calculés à l'aide de l'expression suivante :

$$
D=\frac{D_{N}}{0.84}
$$

$\mathrm{D}$ : diamètre passant de tamisage 


\section{V̀mes Journées Nationales Génie Côtier - Génie Civil \\ Toulon, 29 juin au $1^{\text {er }}$ juillet 2016}

$\mathrm{D}_{\mathrm{N}}$ : diamètre nominal tel que $M=\rho_{S} D_{N}^{3}$

$\mathrm{M}:$ masse du bloc

$\rho_{S}$ : masse volumique des enrochements de filtre, égale à $2750 \mathrm{~kg} / \mathrm{m}^{3}$ sur le site.

Les masses et diamètres caractéristiques des couches successives sont détaillés au tableau 1.

Tableau 1. Masses et diamètres caractéristiques des couches d'enrochements + filtre.

\begin{tabular}{|c|c|c|c|c|c|c|c|c|c|}
\hline \multicolumn{10}{|c|}{ Enrochements $3 t / 5 t$} \\
\hline$E L L$ & $N L L$ & M15 & Mem II & M50 & Mem ul & M60 & NUL & M85 & $E U L$ \\
\hline$y<5 \%$ & $0 \%<y<10 \%$ & $\approx 15 \%$ & & $\approx 50 \%$ & & $\approx 60 \%$ & $70 \%<y<100 \%$ & $\approx 85 \%$ & $y>97 \%$ \\
\hline \multirow[t]{2}{*}{$1750 \mathrm{~kg}$} & $3000 \mathrm{~kg}$ & $3260 \mathrm{~kg}$ & $3850 \mathrm{~kg}$ & $4100 \mathrm{~kg}$ & $4300 \mathrm{~kg}$ & $4700 \mathrm{~kg}$ & $5000 \mathrm{~kg}$ & $7350 \mathrm{~kg}$ & $8250 \mathrm{~kg}$ \\
\hline & D10 & D15 & & D50 & & $D 60$ & & D85 & \\
\hline $1.02 \mathrm{~m}$ & $1.23 \mathrm{~m}$ & $1.26 \mathrm{~m}$ & $1.33 \mathrm{~m}$ & $1.36 \mathrm{~m}$ & $1.38 \mathrm{~m}$ & $1.42 \mathrm{~m}$ & $1.45 \mathrm{~m}$ & $1.65 \mathrm{~m}$ & $1.72 \mathrm{~m}$ \\
\hline \multicolumn{10}{|c|}{ Enrochements $200 \mathrm{~kg} / 1 \mathrm{t}$} \\
\hline$E L L$ & $N L L$ & M15 & Mem Il & M50 & Mem ul & M60 & $N U L$ & M85 & $E U L$ \\
\hline$y<5 \%$ & $0 \%<y<10 \%$ & $\approx 15 \%$ & & $\approx 50 \%$ & & $\approx 60 \%$ & $70 \%<y<100 \%$ & $\approx 85 \%$ & $y>97 \%$ \\
\hline \multirow[t]{2}{*}{$120 \mathrm{~kg}$} & $200 \mathrm{~kg}$ & $270 \mathrm{~kg}$ & $430 \mathrm{~kg}$ & $500 \mathrm{~kg}$ & $570 \mathrm{~kg}$ & $670 \mathrm{~kg}$ & $1000 \mathrm{~kg}$ & $1150 \mathrm{~kg}$ & $1400 \mathrm{~kg}$ \\
\hline & D10 & D15 & & D50 & & $D 60$ & & D85 & \\
\hline $0.42 m$ & $0.50 \mathrm{~m}$ & $0.55 \mathrm{~m}$ & $0.64 \mathrm{~m}$ & $0.67 \mathrm{~m}$ & $0.70 \mathrm{~m}$ & $0.74 \mathrm{~m}$ & $0.85 \mathrm{~m}$ & $0.89 \mathrm{~m}$ & $0.95 \mathrm{~m}$ \\
\hline \multicolumn{10}{|c|}{ Filtre 2/150 mm } \\
\hline$E L L$ & $N L L$ & M15 & M30 & M40 & M50 & M60 & $N U L$ & M85 & $E U L$ \\
\hline$y<5 \%$ & $0 \%<y<10 \%$ & $\approx 15 \%$ & $\approx 30 \%$ & $\approx 40 \%$ & $\approx 50 \%$ & $\approx 60 \%$ & $70 \%<y<100 \%$ & $\approx 85 \%$ & $y>97 \%$ \\
\hline \multirow[t]{2}{*}{$0.000 \mathrm{~kg}$} & $0.000 \mathrm{~kg}$ & $0.000 \mathrm{~kg}$ & $0.000 \mathrm{~kg}$ & $0.003 \mathrm{~kg}$ & $0.025 \mathrm{~kg}$ & $0.148 \mathrm{~kg}$ & $1.627 \mathrm{~kg}$ & $5.493 \mathrm{~kg}$ & $13.020 \mathrm{~kg}$ \\
\hline & D10 & D15 & D30 & D40 & D50 & D60 & & D85 & \\
\hline $0 \mathrm{~mm}$ & $1 \mathrm{~mm}$ & $2 \mathrm{~mm}$ & $6 \mathrm{~mm}$ & $12 \mathrm{~mm}$ & $25 \mathrm{~mm}$ & $45 \mathrm{~mm}$ & $100 \mathrm{~mm}$ & $150 \mathrm{~mm}$ & $200 \mathrm{~mm}$ \\
\hline
\end{tabular}

Les caractéristiques principales sont conformes aux exigences du cahier des charges. Quelques caractéristiques intermédiaires ont été interpolées en nous référant à la courbe de distribution de Rosin-Rammler.

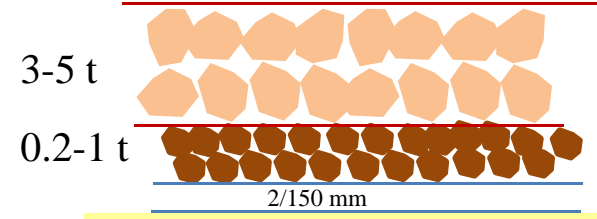

Figure 4. Couches d'enrochements + filtre 2-150 mm sur sable fin.

La granulométrie étendue $\left(D_{15}-D_{50}-D_{85}\right)=(2-25-150 \mathrm{~mm})$ de la couche filtre permet de respecter les critères de rétention aux interfaces [sable fin et filtre 2-150 mm] et [filtre 2-150 mm et 0.2-1 t], mais les grains sont susceptibles de migrer à l'intérieur de la couche filtre, car le critère de stabilité interne $D_{60} / D_{10}<10$ n'est pas respecté. L'application de la formule d'Hoffmans (4) indique qu'il faudrait $3.2 \mathrm{~m}$ d'épaisseur 


\section{Thème 4-Ouvrages portuaires, offshore et de plaisance}

d'enrochement $0.2-1 \mathrm{t}$ pour que le filtre $2-150 \mathrm{~mm}$ soit aussi stable que les enrochements 0.2-1 t eux-mêmes. Cette épaisseur n'étant pas présente, notamment en phase travaux, il faut s'attendre à une perte des matériaux les plus fins, qui risquent de remonter et se perdre dans les blocs $0.2-1$ t en cas de sollicitions hydrodynamiques.

Cette perte de matériaux fins engendre une modification de la composition du filtre, d'abord dans sa partie supérieure. Le filtre devient de plus en plus cru au fur et à mesure que les grains les plus petits disparaissent. La migration des matériaux va s'arrêter naturellement lorsque la granulométrie du filtre, dépourvu de matériaux fins, permettra de respecter le critère de stabilité interne. Dans le cas présent, on montre que cette stabilité est atteinte dès que les grains de diamètre plus petits que $3 \mathrm{~mm}$ à $4 \mathrm{~mm}$ ont été extraits du filtre.

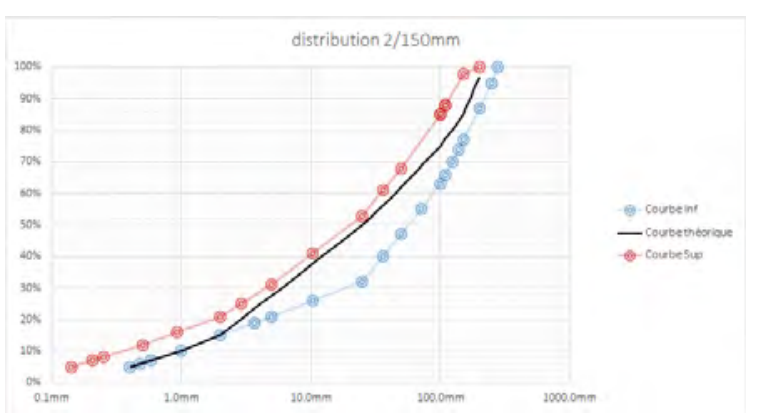

Figure 5. Fuseau granulométrique à l'état initial.

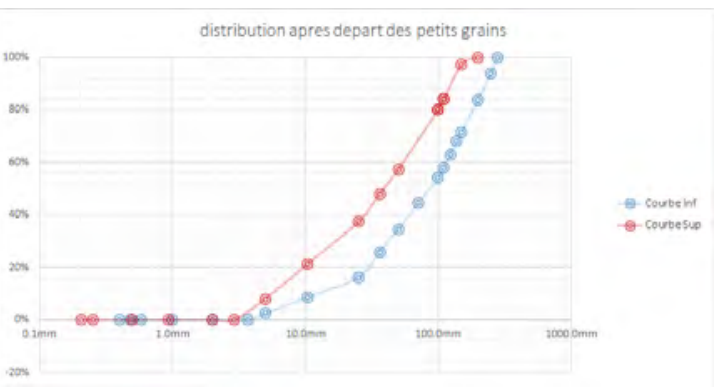

Figure 6. Fuseau granulométrique après la perte des petits matériaux.

Par exemple, la limite crue du fuseau granulométrique enveloppant la courbe théorique (courbe Inf. en bleu sur les graphes ci-dessus) présente initialement les caractéristiques détaillées suivantes :

- $D_{5}=0.4 \mathrm{~mm} ; D_{10}=1.0 \mathrm{~mm} ; D_{15}=2.0 \mathrm{~mm} ; D_{20}=4.3 \mathrm{~mm} ; D_{30}=19 \mathrm{~mm} ; D_{40}=36 \mathrm{~mm}$; $D_{50}=57 \mathrm{~mm} ; D_{60}=88 \mathrm{~mm} ; D_{75}=142 \mathrm{~mm} ; D_{85}=189 \mathrm{~mm} ; D_{97}=262 \mathrm{~mm}$;

- $D_{10} / D_{5}=2.5 ; D_{20} / D_{10}=4.3 ; D_{30} / D_{15}=9.3 ; D_{40} / D_{20}=8.4 ; D_{60} / D_{10}=87.8$.

Après le départ des petits grains $\mathrm{D}<4 \mathrm{~mm}$, qui représentent environ $19 \%$ du volume initial, les plus gros grains restants se répartissent de la façon suivante, respectant le critère de stabilité interne :

- $D_{5}=6.7 \mathrm{~mm} ; \quad D_{10}=12 \mathrm{~mm} ; \quad D_{15}=22 \mathrm{~mm} ; \quad D_{20}=29 \mathrm{~mm} ; \quad D_{30}=42 \mathrm{~mm} ; \quad D_{40}=60 \mathrm{~mm}$; $D_{50}=86 \mathrm{~mm} ; D_{60}=115 \mathrm{~mm} ; D_{75}=162 \mathrm{~mm} ; D_{85}=205 \mathrm{~mm} ; D_{97}=265 \mathrm{~mm}$;

- $D_{10} / D_{5}=1.8 ; D_{20} / D_{10}=2.4 ; D_{30} / D_{15}=1.9 ; D_{40} / D_{20}=2.1 ; D_{60} / D_{10}=9.4$.

Le même calcul peut être effectué pour la limite sableuse du fuseau granulométrique (courbe Sup. en rouge), aboutissant également au départ des grains les plus fins D $<4 \mathrm{~mm}$, représentant $25 \%$ de la composition initiale.

La couche initiale 2-150 mm se scinde naturellement en deux couches schématiques. La couche du haut devient plus crue, telle que $\mathrm{D}_{15}-\mathrm{D}_{85} \approx 12-170 \mathrm{~mm}$ (vers le centre du 


\section{XIV èmes Journées Nationales Génie Côtier - Génie Civil \\ Toulon, 29 juin au $1^{\text {er }}$ juillet 2016}

fuseau), après avoir perdu ses plus petits grains. La perte des grains est progressive au fur et à mesure que l'on pénètre dans le filtre, le départ des petits grains étant majoré par le respect du critère de stabilité interne, lequel occasionne une perte maximale de $19 \%$ à $25 \%$ du volume initial. La couche du bas reste telle quelle, à condition qu'il y ait suffisamment d'épaisseur de filtre 12-170 mm, dépourvu de petits grains, pour la fermer dynamiquement.

L'application de la formule d'Hoffmans sur la partie la limite crue du fuseau granulométrique indique :

- une épaisseur minimale restante de $16 \mathrm{~cm}$ du filtre $\left(\mathrm{D}_{15}-\mathrm{D}_{50}-\mathrm{D}_{85}\right)=(22-86-205 \mathrm{~mm})$ pour que la couche de base $\left(\mathrm{D}_{15}-\mathrm{D}_{50}-\mathrm{D}_{85}\right)=(2-25-150 \mathrm{~mm})$ soit aussi stable que les enrochements 3-5 t, composant le pied en phase définitive de l'ouvrage et couvrant $1.3 \mathrm{~m}$ d'enrochements 0.2-1 t ;

- une épaisseur minimale restante de $25 \mathrm{~cm}$ du filtre $\left(\mathrm{D}_{15}-\mathrm{D}_{50}-\mathrm{D}_{85}\right)=(22-86-205 \mathrm{~mm})$ pour que la couche de base $\left(D_{15}-D_{50}-D_{85}\right)=(2-25-150 \mathrm{~mm})$ soit aussi stable que les enrochements $0.2-1 \mathrm{t}$ en phase de travaux.

La perte du matériau filtre étant de 19\% (limite crue du fuseau), l'épaisseur initiale de matériau impactée par la fuite potentielle de petits grains est telle que :

- Epaisseur initiale=16 cm à $25 \mathrm{~cm} /(1-0.19)$, soit $20 \mathrm{~cm}$ à $31 \mathrm{~cm}$

- Perte associée $=20 \mathrm{~cm}$ à $31 \mathrm{~cm} \times 0.19$, soit $4 \mathrm{~cm}$ à $6 \mathrm{~cm}$

Une pose initiale sur $60 \mathrm{~cm}$ de filtre 2-150 mm permet donc d'obtenir, à terme :

- $60 \mathrm{~cm}$ (initial) - [16-25 cm] (filtre cru 12-170 mm) - [4-6 cm] (départ petits grains $)=[29-40 \mathrm{~cm}]$ de couche filtre dynamiquement stable, offrant une rétention géométrique au sable fin.

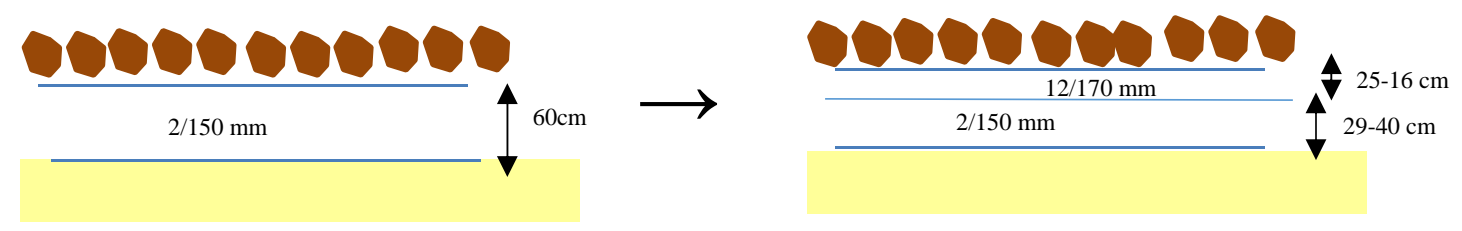

Figure 7. Vue schématique de l'évolution naturelle de la granulométrie du filtre.

La perte d'épaisseur de 4 à $6 \mathrm{~cm}$ suppose que le départ des petits grains entraîne un tassement proportionnel à la perte du volume de matériaux. En réalité, le fuite des petits grains laisse place à un peu plus de vide, augmentant la porosité, et baissant l'épaisseur de tassement estimée.

La même approche sur la partie sableuse du fuseau entraîne certes un départ de 25\% des grains, mais une épaisseur de filtre impacté d'au maximum $18 \mathrm{~cm}$ (en phase travaux), avec moins de $5 \mathrm{~cm}$ de tassement.

Les courbes Inf. (en bleu) et Sup. (en rouge) sont déterminées par itérations successives en se fixant comme contrainte de disposer d'au moins $30 \mathrm{~cm}$ de matériaux filtres non impactés par le départ de grains, et de limiter les tassements à quelques centimètres. On 
s'astreint également à tracer une courbe granulométrique monotone croissante sur l'échelle logarithmique, caractéristique des compositions dites autostables.

Suivant la même approche théorique, d'autres adaptations sont possibles, comme la pose d'un filtre initialement un plus cru, ne fermant pas géométriquement, mais dynamiquement le sable fin. Une épaisseur de $80 \mathrm{~cm}$ permettrait une fermeture dynamique, avec un coefficient de sécurité respectable sur les facteurs de réduction de contraintes de cisaillement. Même si la distribution granulométrique réelle en sortie de carrière tend à se rapprocher de la distribution théorique étudiée, fermant géométriquement le sable, une épaisseur minimale locale de $80 \mathrm{~cm}$ (soit en moyenne au moins $1 \mathrm{~m}$ ) a été imposée pour augmenter les coefficients de sécurité.

\section{Conclusion}

Les avancées théoriques et expérimentales récentes en matière de fermeture dynamique de filtres granulaires géométriquement ouverts ont été exploitées pour justifier de la conception d'une adaptation par rapport à une solution de type géotextile. Les règles classiques de fermeture géométrique par succession de plusieurs couches auraient été difficilement mises en œuvre au réel, tandis que la solution monocouche épaisse proposée a été effectivement réalisée sur site. La touche originale développée ici consiste à exploiter les caractéristiques granulométriques vers lesquelles un filtre initialement ouvert tend, au fur et à mesure qu'il perd ses grains les plus fins. La méthode doit toutefois être appliquée en retenant les valeurs hautes sécurisantes des paramètres, le sujet pouvant encore faire l'objet de nombreuses recherches complémentaires.

\section{Remerciements :}

L'auteur remercie Pierre Aristaghes, Directeur Général d'EGIS-Ports, qui a contribué à orienter la solution originale, ainsi que Stéphane Magné et Nicolas Thébaud du groupement d'Entreprises GTOI- SBTPC-VINCI.

\section{Références bibliographiques}

HOFFMANS G.J.C.M. (2012). The influence of Turbulence on Scour and Erosion. Deltares Select Series, Delft.

VAN DE SANDE S. (2013). Stability of open filter structures. Msc Thesis June 2012, Delft, Revision August 2013.

VERHEIJ H., HOFFMANS G., DORST K., VAN DE SANDE S. (2012). Interface stability of granular filter structures under currents. ICSE6-225, Paris August 27-31. 